\title{
Western Blot Seroindeterminate Individuals for Human T-lymphotropic Virus 1/2 (HTLV-1/2) in Fortaleza (Brazil): A Serological and Molecular Diagnostic and Epidemiological Approach
}

Terezinha de Jesus Teixeira Santos, Carlos Maurício de Castro Costa, Patrick Goubau,Anne-Mieke Vandamme, Jan Desmyter, Sonia Van Dooren, Rosa M. S. Mota, Francine Bovy de Castro Costa, Ana C.S. Oliveira, Vânia Barreto A.F. Gomes, Anna B. Carneiro-Proietti, Veralice Meireles Sales de Bruin, Francisca C. F. de Sousa and Reinaldo Barreto Oriá

\author{
Department of Physiology and Pharmacology, \\ Federal University of Ceará, Fortaleza, CE, Brazil; \\ Unit of Virology, Université Catholique de Louvain, \\ Louvain, Belgium; Rega Institute for Medical \\ Research, Katholieke Universiteit Leuven, Leuven, \\ Belgium; Department of Statistics and Applied \\ Mathematics, Federal University of Ceará, \\ Fortaleza, CE, Brazil; HEMOCE of Fortaleza, \\ Fortaleza, CE, Brazil; HEMOMINAS Foundation, \\ Belo Horizonte, $M G$, Brazil
}

\begin{abstract}
How to handle Western blot (WB) seroindeterminate individuals for Human T-lymphotropic Virus 1/2 (HTLV-1/2) constitutes a challenge for blood banks and families. We made a crosssectional study of 191 enzyme linked immunoassay (EIA) reactive individuals from the hematological center (HEMOCE) of Fortaleza (Brazil), examining their serological (WB) and molecular (PCR) diagnosis, and demographic profiles, as well as a possible association of their condition with other infectious pathologies and risk factors. Ethical institutional approval and personal consent were obtained. Out of 191 EIA reactive individuals, 118 were WB seroindeterminate and 73 were seropositive for HTLV-1/2. In the PCR analysis of $41 \mathrm{WB}$ seroindeterminate individuals, $9(22 \%)$ were positive and $32(78 \%)$ were negative for HTLV-1/2. The demographic analysis indicated a trend towards a predominance of males among the seroindeterminate individuals and females in the seropositive ones. The seroindeterminate individuals were younger than the seropositive ones. We did not find any association of these conditions with syphilis, Chagas disease or HIV or hepatitis, and with risk factors such as breast-feeding, blood transfusion, STD (syphilis) and IDU.

Key Words: Epidemiology, PCR, HTLV-1/2, seroindeterminate individuals, hepatitis, syphilis, blood transfusion.
\end{abstract}

The human T-lymphotropic virus (HTLV) belongs to the family Retroviridae and the subfamily Oncovirinae. It is divided into types 1 and 2, sharing around $60 \%$ similarity. Based on molecular epidemiology HTLV-1 is

Received on 09 July 2002; revised 30 September 2002.

Address for correspondence: Dr. Terezinha de Jesus Teixeira Santos - Lab. de Neurologia Experimental e NeurofisiologiaDepto. de Fisiologia e Farmacologia - Universidade Federal do Ceará - Rua Cel. Nunes de Melo, 1127 - Rodolfo Teófilo Fortaleza-CE-60.430-270-Fax: +55 85 288-8333-E-mail: mcastro@ufc.br

The Brazilian Journal of Infectious Diseases 2003;7(3):202-209 (C) 2003 by The Brazilian Journal of Infectious Diseases and Contexto Publishing. All rights reserved. divided into the 1a (cosmopolitan), $1 \mathrm{~b}$ (Central Africa), 1c (Melanesia), 1d, 1e and 1f (Central Africa) subtypes, and HTLV-2 into the 2a, $2 \mathrm{~b}$ and $2 \mathrm{~d}$ subtypes. HTLV-1/ 2 is variably endemic in Central and West Africa, the Caribbean, South America, Japan and Melanesia. In Brazil, its prevalence is $0.46 \%$ [1]. This retrovirus is transmitted vertically (mother-to-child) and horizontally (sexual contact, blood transfusion and intravenous drug use). Tropical Spastic Paraparesis/HTLV-1associated Myelopathy (TSP/HAM) and Adult T-cell Leukemia/Lymphoma (ATL) are the main associated pathologies [2,3].

The laboratorial diagnosis of HTLV is performed using screening (enzyme linked immunoassay (EIA) and 
particle agglutination), confirmatory (Western blot, IFA, RIPA, and more recently INNO-LIA) and molecular (PCR, NASBA and bDNA) tests. Serological tests for HTLV infection have been mandatory in some countries since 1986, and in Brazil since 1993. Screening and confirmatory results from blood banks are varied, with negative, positive and indeterminate profiles apparent.

Blood banks worldwide are thus faced with blood donor candidates exhibiting a seroindeterminate Western blot $(\mathrm{WB})$ profile. This is mainly reported in tropical and in some temperate regions endemic for HTLV-1/2 infection, and less frequently in northern countries, where prevalence of HTLV-1/2 is very low [4,5].

Individuals seroindeterminate for HTLV-1/2 represent a challenge for blood banks, as well as in familial contexts, due to the risk they represent in spreading infections through blood donation and sexual contact, if truly infected, and because of the psychological burden it presents for the person with this undefined condition.

With the aim of delineating a profile of Western Blot (WB) seroindeterminate individuals for HTLV-1/2 from the hematological center (HEMOCE) of Fortaleza (Brazil), we carried out a cross-sectional study, taking into consideration their serological and molecular diagnosis, and demographic characteristics, as well as the possible association of this condition with other infectious pathologies and risk factors.

\section{Materials and Methods}

One hundred and ninety one EIA reactive candidates among blood donors at the HEMOCE were tested with confirmatory HTLV WB version 2.4 (WB2.4) from Genelabs Diagnostic. Seropositivity was interpreted according to the stringent criteria indicated by Genelabs Diagnostics. A WB test was scored as HTLV-1 positive only if bands for the gag proteins ( $\mathrm{p} 19$ with or without $\mathrm{p} 24$ ) and two env proteins (GD21 and rgp46-1) were present. It was scored as HTLV-2 positive if bands for the gag proteins (p24 with or without p19) and two env proteins (GD21 and rgp46-2) were found. The test was considered as indeterminate if specific bands for HTLV that did not fulfill the criteria of positivity for HTLV-1 and 2 were present. Those that did not exhibit bands specific for HTLV were considered negative.

For HTLV-1/2 molecular analysis, 41 out of the 118 samples from WB seroindeterminate individuals were tested with PCR at the Rega Institute for Medical Research and University Hospitals of the Katholieke Universiteit Leuven(Belgium).

For provirus detection in patient samples, $10^{6}$ peripheral blood mononuclear cells were pelleted and lysed in PCR buffer (10 mM Tris-HCl, pH 8.3, 50 $\mathrm{mM} \mathrm{KCl}$ Perkin-Elmer), containing $2 \mathrm{mM} \mathrm{MgCl}_{2}$, $0.5 \%$ Tween-20, $0.5 \%$ NP40, and $100 \mu \mathrm{g} / \mathrm{ml}$ proteinase K (Boehringer Mannheim, stabilized proteinase $\mathrm{K}$ solution), for $1 \mathrm{hr}$ at $56^{\circ} \mathrm{C}$. The DNA was extracted using phenol/chloroform (Life Technologies), precipitated with ethanol, and dissolved in Milli-Q water (Millipore system). The Qiagen blood kit was used (Westburg) for extracting the DNA of the control cell lines.

DNA from $10^{5}$ cells was used for amplification in a $50 \mu \mathrm{l}$ reaction volume containing $10 \mathrm{mM}$ Tris- $\mathrm{HCl}$, $\mathrm{pH} 8.3,50 \mathrm{mM} \mathrm{KCl}, 200 \mu \mathrm{M}$ nucleotide triphosphates, $0.2 \mu \mathrm{M}$ outer or $0.5 \mu \mathrm{M}$ inner primers and 00.25 U/ $\mu$ l AmpliTaq (Perkin-Elmer), with 1.5 $\mathrm{mM} \mathrm{MgCl}$ for the generic primers and $2 \mathrm{mM}$ for the discriminatory inner primers (Table 1). Cycling conditions on a GeneAmp PCR System 9600 (PerkinElmer) were $30 \mathrm{sec} 94^{\circ} \mathrm{C}, 15 \mathrm{sec} 50^{\circ} \mathrm{C}, 45 \mathrm{sec} 72^{\circ} \mathrm{C}$ for the outer generic PCR; on a Triothermobloc (Biometra) they were $30 \mathrm{sec} 95^{\circ} \mathrm{C}, 15 \mathrm{sec} 52^{\circ} \mathrm{C}, 1$ $\min 72^{\circ} \mathrm{C}$ for the inner generic PCR; and $30 \sec 95^{\circ} \mathrm{C}$, $15 \mathrm{sec} 50^{\circ} \mathrm{C}, 1 \mathrm{~min} 72^{\circ} \mathrm{C}$ for the inner discriminatory PCR. The outer fragment was amplified for 45 cycles; $2 \mu \mathrm{l}$ was transferred to the inner PCR, and amplified for 25 cycles. A globin PCR was performed using primer pair PC03/KM38 to assure the quality of the DNA [6]. Amplification products were separated on a $6 \%$ polyacrylamide gel and visualized by ethidium bromide staining. The images were processed on a video imager (ImageMaster VDS, Pharmacia Biotech, Roosendaal, The Netherlands). Positive controls for the PCR were MT-2 cells harboring 
HTLV-1 MT2 (kindly provided by Luc Montaigner, Institut Pasteur, Paris, France), SI-5 cells harboring HTLV-1 Mel5 (kindly provided by Richard Yanagihara, NIH, Bethesda, MD), 729 cells harboring HTLV-2 Mo (kindly provided by Helen Lee, Abbott Laboratories, North Chicago, IL) clone 19 cells harboring HTLV-2 (kindly provided by Dana Gallo, Viral and Rickettsial Disease Laboratory, Berkeley, CA), Cu cells harboring HTLV-2, Gu, PH969 cells harboring STLV-PH969, and PP1664 cells harboring STLV-PP1664. Negative controls for the PCR were Hut-78 cells (kindly provided by Institut Pasteur, Brussels, Belgium) and ACH-2 cells (kindly provided through the AIDS reagent project of the Medical Research Council, UK).

The PCR primers were designed using the tax gene alignment of all available HTLV and STLV strains. The primer sequences for the generic nested PCR and the four discriminatory inner PCRs are given in Table 1. Primers were developed and analyzed using the Oligo software (Medprobe, Oslo, Norway). Synthesis was carried out by Pharmacia Biotech and Perkin-Elmer/Applied Biosystems.

For the demographic study, age and gender were considered. Thus, 118 seroindeterminate and 73 seropositive individuals previously diagnosed by WB, and 41 out of $118 \mathrm{WB}$ seroindeterminate individuals diagnosed by PCR, were included.

The analysis of co-infection and risk factors was based on previous information obtained from some of the individuals of our series. This explains the different numbers shown below.

The analysis of co-infection included laboratorial evidence for infections such as syphilis, Chagas disease, HIV, and hepatitis. Thus, $83 \mathrm{WB}$ seroindeterminate and 27 seropositive individuals for HTLV-1/2, as well as $31 \mathrm{WB}$ seroindeterminate individuals, diagnosed by PCR, were studied.

The risk factors, breast-feeding, blood transfusion, sexually transmitted diseases (STD) (syphilis) and intravenous drug use (IDU) were analyzed in 95 seroindeterminate and 65 seropositive individuals diagnosed by WB, and in 36 seroindeterminate ones diagnosed by PCR.
This research project was approved by the Committee for Ethics in Research of the Faculty of Medicine of the Federal University of Ceará (Brazil), with the participating individuals each signing a consent document.

Descriptive statistics and association tests for contingency tables (Fischer's exact test) were used in the analysis. Levene's and Student's t-tests were used for analysis of variance and to determine if the means differed [7]. A level of significance of $p \leq 0.05$ was considered.

\section{Results}

The WB confirmatory test of our sample of 191 EIA reactive individuals showed that 73 were seropositive and 118 were seroindeterminate for HTLV-1/2.

The PCR analysis of the $41 \mathrm{WB}$ seroindeterminate individuals revealed that $32(78 \%)$ were negative and 9 ( $22 \%$ ) were positive ( 7 for HTLV-1 and 2 for HTLV-2).

The demographic analysis of the $118 \mathrm{WB}$ seroindeterminate and 73 seropositive individuals showed that there was a significant $(p=0.0420)$ association between sex and WB results, from which one could depict a trend for female predominance in the group of individuals seropositive for HTLV-1/2, and for male predominance in the group of seroindeterminate individuals (Table 2). The mean age of HTLV-1/2 seroindeterminate and seropositive individuals was 34.5 years $(\mathrm{SD}=9.6$; Median=32) and 40.4 years $(S D=14.7$; Median $=41.5)$, respectively. The mean age of seroindeterminate individuals was significantly lower $(p=0.0029)$ than that of HTLV-1/2 seropositive individuals .

When we examined associated diseases, there was no significant correlation between the results of WB and PCR tests and co-infections (syphilis, Chagas disease, HIV, and hepatitis) (Tables 3 and 4).

There was also no significant association of WB HTLV-1/2 seroindeterminate and seropositive individuals with risk factors such as breast-feeding, blood transfusion, STD (syphilis) and IDU, based on the WB and $\mathrm{PCR} / \mathrm{WB}$ results (Table 5). 
Table 1. Primers in the tax/rex gene used for the generic and discriminatory HTLV PCRs

\begin{tabular}{llll}
\hline PCR & Primers & \multicolumn{1}{c}{ Sequence 5'-3' } & \multicolumn{1}{c}{ Orientation } \\
\hline Generic outer & AV45 & GGACGCGTT(A/G)TC(A/G)GCTC & Sense \\
& AV46 & (G/T)GG(A/G)GAIAG(C/T)TGGTA(G/T)AGGTA & Antisense \\
Generic inner & AV42 & CTCCCCTCCTTCCCCAC & Sense \\
& AV43 & CCA(G/C)(A/G)(G/T)GGTGTAIAIGTTTTGG & Antisense \\
HTLV-1 inner & AV49 & CCCTCCTTCCTCCAGGCCAT & Sense \\
& AV80 & GGTCTGGAAAAGACAGGGTTG & Antisense \\
HTLV-2 inner & AV50 & TCAATCAATGCGAAAGCACACC & Sense \\
& AV81 & TAGGTATAGGCATACTACGGTT & Antisense \\
STLV-PH969 inner & AV51 & ACAATTGCCTCGAGCTCACCC & Sense \\
& AV82 & GAGGCACACGACGGAGCT & Antisense \\
STVL-PP1664 inner & AV52 & ACGGGTGCCTATACCCAACTC & Sense \\
& AV83 & GGTACAAGCAAACTACGGTTC & Antisense \\
\hline
\end{tabular}

Table 2. Distribution of WB seroindeterminate $(n=118)$ and seropositive $(n=73)$ individuals by gender

\begin{tabular}{|c|c|c|c|c|c|c|}
\hline \multirow[t]{3}{*}{ WB Test } & \multicolumn{4}{|c|}{ Sex } & & \\
\hline & \multicolumn{2}{|c|}{ Female } & \multicolumn{2}{|c|}{ Male } & \multicolumn{2}{|c|}{ Total } \\
\hline & $\mathbf{n}$ & $\%$ & $\mathbf{n}$ & $\%$ & $\mathbf{n}$ & $\%$ \\
\hline Seroindeterminate $(\mathrm{n}=118)$ & 33 & 28.0 & 85 & 72.0 & 118 & 100.0 \\
\hline Seropositive $(n=73)$ & 31 & 42.5 & 42 & 57.5 & 73 & 100.0 \\
\hline
\end{tabular}

\section{Discussion}

We found that $9(22 \%)$ and $32(78 \%)$ of the 41 WB seroindeterminate individuals were positive and negative for HTLV-1/2, respectively, as determined by PCR; consequently, this method could be useful for defining the condition of the WB seroindeterminate individuals for HTLV-1/2.

These findings are coherent with previous investigations. For example, Medrano et al. [8] studied 16 seroindeterminate individuals, and found HTLV-1/ 2 with PCR in 4 (25\%). In addition, Garin et al. [9] studied 67 blood samples of seroindeterminate individuals in Zaire, and he found HTLV-1 (with PCR) in only $10(15 \%)$ of them, whilst none were positive for HTLV-2. On the other hand, Caterino-de-Araújo et al. [10] analyzed 553 samples from HIV positive individuals from São Paulo (Brazil), of which 24(4.3\%) were seroindeterminate for HTLV-1/2, 25\% of them being positive for HTLV-2 by PCR. Delaporte et al. [11] carried out two studies in Gabon and found positivity by PCR in $16 \%$ and $31 \%$ of the WB seroindeterminate samples. However, Lipka et al. [12] analyzed 67 seroindeterminate samples and did not find positivity for HTLV-1/2 by PCR in any of them. Nerurkar et al. [13] studied 27 seroindeterminate individuals from Papua New Guinea and the Salomon Islands, and viral sequences of HTLV-1 were not found in any of them. Gessain et al. [14] reported a high prevalence of seroindeterminate patterns in the tropics, with PCR negative results. This may mean that there are additional etiologies for this condition. 
Table 3. Distribution of WB seroindeterminate $(n=83)$ and seropositive $(n=27)$ individuals for HTLV-1/2 as a function of the Co-Infection test results

\begin{tabular}{|c|c|c|c|c|c|c|c|c|c|c|c|c|}
\hline \multirow[t]{4}{*}{ WB Test } & \multicolumn{12}{|c|}{ Co-Infection Test Results } \\
\hline & \multicolumn{4}{|c|}{ Chagas disease } & \multicolumn{4}{|c|}{ Syphilis } & \multicolumn{4}{|c|}{ HIV } \\
\hline & \multicolumn{2}{|c|}{ Negative } & \multicolumn{2}{|c|}{ Positive } & \multicolumn{2}{|c|}{ Negative } & \multicolumn{2}{|c|}{ Positive } & \multicolumn{2}{|c|}{ Negative } & \multicolumn{2}{|c|}{ Positive } \\
\hline & $\mathbf{n}$ & $\%$ & $\mathbf{n}$ & $\%$ & $\mathbf{n}$ & $\%$ & $\mathbf{n}$ & $\%$ & $\mathbf{n}$ & $\%$ & $\mathbf{n}$ & $\%$ \\
\hline Seroindeterminate & 83 & 76.1 & - & - & 83 & 75.5 & - & - & 81 & 75.0 & 2 & 100.0 \\
\hline Seropositive & 26 & 23.9 & 1 & 100.0 & 27 & 24.5 & - & - & 27 & 25.0 & - & - \\
\hline Total & 109 & 100.0 & 1 & 100.0 & 110 & 100.0 & - & - & 108 & 100.0 & 2 & 100.0 \\
\hline \multirow[t]{4}{*}{ WB Test } & \multicolumn{12}{|c|}{ Co-Infection Test Results } \\
\hline & \multicolumn{4}{|c|}{ Hepatitis B (HBsAg) } & \multicolumn{4}{|c|}{ Hepatitis B (HBc) } & \multicolumn{4}{|c|}{ Hepatitis C (HCV) } \\
\hline & \multicolumn{2}{|c|}{ Negative } & \multicolumn{2}{|c|}{ Positive } & \multicolumn{2}{|c|}{ Negative } & \multicolumn{2}{|c|}{ Positive } & \multicolumn{2}{|c|}{ Negative } & \multicolumn{2}{|c|}{ Positive } \\
\hline & $\mathbf{n}$ & $\%$ & $\mathbf{n}$ & $\%$ & $\mathbf{n}$ & $\%$ & $\mathbf{n}$ & $\%$ & $\mathbf{n}$ & $\%$ & $\mathbf{n}$ & $\%$ \\
\hline Seroindeterminate & 78 & 75.7 & 5 & 71.4 & 71 & 78.9 & 12 & 57.1 & 78 & 77.2 & 5 & 50.0 \\
\hline Seropositive & 25 & 24.3 & 2 & 28.6 & 19 & 21.1 & 9 & 42.9 & 23 & 22.8 & 5 & 50.0 \\
\hline Total & 103 & 100.0 & 7 & 100.0 & 90 & 100.0 & 21 & 100.0 & 101 & 100.0 & 10 & 100.0 \\
\hline
\end{tabular}

Table 4. Distribution of PCR negative $(n=25)$ and PCR/WB positive $(n=33)$ individuals for HTLV-1/2 as a function of the Co-Infection test results

\begin{tabular}{|c|c|c|c|c|c|c|c|c|c|c|c|c|}
\hline \multirow[t]{4}{*}{ PCR/WB Test } & \multicolumn{12}{|c|}{ Co-Infection Test Results } \\
\hline & \multicolumn{4}{|c|}{ Chagas Disease } & \multicolumn{4}{|c|}{ Syphilis } & \multicolumn{4}{|c|}{ HIV } \\
\hline & \multicolumn{2}{|c|}{ Negative } & \multicolumn{2}{|c|}{ Positive } & \multicolumn{2}{|c|}{ Negative } & \multicolumn{2}{|c|}{ Positive } & \multicolumn{2}{|c|}{ Negative } & \multicolumn{2}{|c|}{ Positive } \\
\hline & $\mathrm{n}$ & $\%$ & $\mathrm{n}$ & $\%$ & $\mathrm{n}$ & $\%$ & $\mathrm{n}$ & $\%$ & $\mathrm{n}$ & $\%$ & $\mathrm{n}$ & $\%$ \\
\hline Negative (PCR) & 25 & 43.9 & - & - & 25 & 43.1 & - & - & 24 & 42.1 & 1 & 100.0 \\
\hline $\begin{array}{l}\text { Positive HTLV-1 } \\
\text { (PCR/WB) }\end{array}$ & 19 & 33.3 & - & - & 19 & 32.8 & - & - & 19 & 33.3 & - & - \\
\hline $\begin{array}{l}\text { Positive HTLV-2 } \\
\text { (PCR/WB) }\end{array}$ & 13 & 22.8 & 1 & 100.0 & 14 & 24.1 & - & - & 14 & 24.6 & - & - \\
\hline Total & 57 & 100.0 & 1 & 100.0 & 58 & 100.0 & - & - & 57 & 100.0 & 1 & 100.0 \\
\hline \multirow[t]{4}{*}{ PCR/WB Test } & \multicolumn{12}{|c|}{ Co-infection Test Results } \\
\hline & \multicolumn{4}{|c|}{ Hepatitis B (HBsAg) } & \multicolumn{4}{|c|}{ Hepatitis B (HBc) } & \multicolumn{4}{|c|}{ Hepatitis C (HCV) } \\
\hline & \multicolumn{2}{|c|}{ Negative } & \multicolumn{2}{|c|}{ Positive } & \multicolumn{2}{|c|}{ Negative } & \multicolumn{2}{|c|}{ Positive } & \multicolumn{2}{|c|}{ Negative } & \multicolumn{2}{|c|}{ Positive } \\
\hline & $\mathbf{n}$ & $\%$ & $\mathbf{n}$ & $\%$ & $\mathbf{n}$ & $\%$ & $\mathbf{n}$ & $\%$ & $\mathbf{n}$ & $\%$ & $\mathbf{n}$ & $\%$ \\
\hline Negative (PCR) & 23 & 42.6 & 2 & 50.0 & 21 & 47.7 & 4 & 26.7 & 22 & 44.9 & 3 & 30.0 \\
\hline $\begin{array}{l}\text { Positive HTLV-1 } \\
\text { (PCR/WB) }\end{array}$ & 18 & 33.3 & 1 & 25.0 & 13 & 29.5 & 6 & 40.0 & 13 & 26.5 & 6 & 60.0 \\
\hline $\begin{array}{l}\text { Positive HTLV-2 } \\
\text { (PCR/WB) }\end{array}$ & 13 & 24.1 & 1 & 25.0 & 10 & 22.7 & 5 & 33.3 & 14 & 28.6 & 1 & 10.0 \\
\hline Total & 54 & 100.0 & 4 & 100.0 & 44 & 100.0 & 15 & 100.0 & 49 & 100.0 & 10 & 100.0 \\
\hline
\end{tabular}


According to data (unpublished) from the hematological center of Fortaleza (HEMOCE-Ceará) from 1997 to 2000 , the prevalence of seroindeterminate individuals was $0.16 \%$ in the general population of blood donors and $21.99 \%$ among the population of EIA reactive individuals. In another study, also in Ceará (Brazil), De Castro-Costa et al. [15] analyzed 1042 blood samples of non-neurological patients from Fortaleza $(n=593)$ and Crato $(n=449)$, of which 6 $(1.0 \%)$ in Fortaleza and $2(0.4 \%)$ in Crato exhibited a seroindeterminate profile.

There is however variation of prevalence depending on the geographic area and the population studied. While in Europe and USA the prevalence is low, in tropical areas it reaches higher values [4,5]. Lal et al. [16] analyzed 267,650 samples from blood donors, among which 379 $(0.14 \%)$ were WB seroindeterminate. Césaire et al. [17] carried out an analysis of 9,759 blood donors from the West Indies and found 39 (0.4\%) seropositive and $49(0.5 \%)$ seroindeterminate individuals for HTLV-1. Mauclère et al. [18] studied 3783 individuals from the rural population of Cameroon and found a prevalence of $1.6 \%$ seroindeterminates. Garin et al. [9] studied the frequency of positivity for HTLV-1/2 in 98 samples of blood from individuals from Zaire and found that $28(28.6 \%)$ were seropositive, $3(3.0 \%)$ were seronegative and $67 \quad(68.4 \%)$ were seroindeterminate. In Brazil, Caterino-de-Araújo et al. [10] investigated HTLV-1/2 infection in 553 samples of serum from HIV patients in São Paulo, of which $24(4.3 \%)$ had an indeterminate profile.

Demographically, young men predominated among the seroindeterminate individuals, while older women predominated among the ones seropositive for HTLV$1 / 2$. The difference in age between seroindeterminate and seropositive individuals could be due to tardive seroconversion; while the trend of women predominating among the seropositive individuals could be due to their greater exposure to infection with HTLV-1/2 [18,19]. Césaire et al. [17], however, did not find significant gender differences between groups seroindeterminate and seropositive for HTLV-1/2, although consistent with our findings, they found that the seroindeterminate and seronegative individuals were younger than the seropositives.

Our investigation of HTLV-1/2 seroindeterminate and seropositive individuals with co-infections revealed seropositive individuals with anti-HBc (hepatitis B) and HCV (hepatitis C) antibodies, which is also shown in supported by previous reports from Brazil $[20,21]$ and from other countries [17,19].

Other presumptive associations of indeterminate profiles have been reported with Plasmodium falciparum [22-27], varicella zoster virus or herpes simplex [28-31], and dengue virus [32]. Moreover, recent seroconversion to positivity for HTLV-1/2 [17], incomplete reactions to molecularly distinct variants of HTLV-1/2 or eventually to another exogenous or endogenous retrovirus [30], and transient infection by HTLV-1 [14], have also been reported. Other conditions are also supposedly correlated with a seroindeterminate profile. For example, Haynes et al. [33] and Palker et al. [34] analyzed the antigenic relationship between $\mathrm{p} 19$ and the thymic epithelium, possibly linked to immune conditions in HTLV-1 infected individuals. Seroindeterminate patterns have been found in Europe and the USA in a series of patients with multiple sclerosis, although at a very low frequency [35]. A seroindeterminate profile may also be associated with hematological abnormalities compatible with viral infection [36] or with a possible, as yet unidentified, genetic or environmental factor [37].

These different possibilities offer an exciting path of research towards the etiology and significance of the seroindeterminate profile for HTLV-1/2 infection, especially in tropical and in some temperate regions. Longitudinal follow-up of these seroindeterminate individuals, defined initially as positive or negative by PCR, is suggested. This follow-up should include other sophisticated tests for defining HTLV-1/2 infection, such as for example, INNO-LIA and real time PCR, as well as tests for defining parasitic, bacterial or viral infections, endemic in the related environmental setting. This may stimulate multicentric studies to define the real status of WB seroindeterminate individuals in these different contexts. 
Table 5. Distribution of WB seroindeterminate $(n=95)$ and seropositive $(n=69)$ individuals for HTLV-1/2 as a function of the Risk Factors

\begin{tabular}{l} 
WB Test \\
\cline { 2 - 6 } \\
\cline { 2 - 6 }
\end{tabular}

\section{References}

1. Galvão-Castro B., Loures L., Rodrigues L.G., et al. Distribution of human T-lymphotropic virus type 1 among blood donors: a nationwide Brazilian study. Transfusion 1997;37(2):242-3.

2. Gessain A., Barin F., Vernant J.C., et al. Antibodies to human T-lymphotropic virus type-1 in patients with tropical spastic paraparesis. Lancet 1985;2:407-10.

3. Osame M., Izuma S., Igata A., et al. Blood transfusion and HTLV-1 associated myelopathy. Lancet 1986; 2 : 104-5.

4. Lal R.B., Lipka J.J., Foung S.K., et al. Human T lymphotropic virus type 1/2 in Lake Lindu Valley, Central Sulawesi, Indonesia. J Acquir Immune Defic Syndr Hum Retrovirol 1993; 6:1067-8.

5. Mahieux R., Horal P., Mauclère P., et al. Human T-cell lymphotropic virus type 1 gag indeterminate Western blot patterns in Central Africa: relationship to Plasmodium falciparum infection. J Clin Microbiol 2000;38:4049-57.

6. Vandamme A.M., Fransen K., Debaisieux L., et al. Standardization of primers and an algorithm for HIV-1 diagnostic PCR evaluated in patients harbouring strains of diverse geographical origin. The Belgian AIDS Reference Laboratories. J Virol Methods 1995;51:305-16.
Risk Factors

\begin{tabular}{|c|c|c|c|c|c|c|c|c|c|}
\hline \multicolumn{3}{|c|}{ Blood transfusion } & \multicolumn{4}{|c|}{ STD (Syphilis) } & \multicolumn{3}{|c|}{ IDU } \\
\hline No & & Yes & & No & & Yes & & No & Yes \\
\hline n $\%$ & $\mathbf{n}$ & $\%$ & $\mathbf{n}$ & $\%$ & $\mathbf{n}$ & $\%$ & $\mathbf{n}$ & $\%$ & n $\%$ \\
\hline 62.1 & 13 & 46.4 & 75 & 63.0 & 20 & 48.8 & 94 & 59.9 & 50.0 \\
\hline 37.9 & 15 & 53.6 & 44 & 37.0 & 21 & 51.2 & 64 & 40.1 & 50.0 \\
\hline 100.0 & 28 & 100.0 & 119 & 100.0 & 41 & 100.0 & 158 & 100.0 & 2100.0 \\
\hline
\end{tabular}

$\mathrm{p}=0.1416$

$\mathrm{p}=0.1402$

$\mathrm{p}=1.0000$

7. Freeman J., Daniel H. Applied categorical data analysis. Marcel Dekker, 1987.

8. Medrano F.J., Soriano V., Calderón E.J., et al. Significance of indeterminate reactivity to human $\mathrm{T}$-cell lymphotropic virus in Western blot analysis of individuals at risk. Eur J Clin Microbiol Infect Dis 1997; 16:249-52.

9. Garin B., Gosselin S., De Thé G., Gessain A. HTLV-1/2 infection in a high viral endemic area of Zaire, Central Africa: comparative evaluation of serology, PCR, and significance of indeterminate Western blot pattern. J Med Virol 1994;44:104-9.

10. Caterino-de-Araújo A., de los Santos-Fortuna E., Meleiro M.C., et al. Sensitivity of two enzyme-linked immunosorbent assay tests in relation to Western blot in detecting human T-cell lymphotropic virus type 1 and 2 infection among HIV-1 infected patients from São Paulo, Brazil. Diagn Microbiol Infect Dis 1998;30:173-82.

11. Delaporte E., Monplaisir N., Louwagie J., et al. Prevalence of HTLV-1 and HTLV-2 infection in Gabon, Africa: comparison of the serological and PCR results. Int J Cancer 1991;49:373-6.

12. Lipka J.J., Young K.K., Kwok S.Y., et al. Significance of human T-lymphotropic virus type 1 indeterminant serological findings among healthy individuals. Vox Sang 1991;61:171-6.

13. Nerurkar V.R., Miller M.A., Leon-Monzon M.E., et al. Failure to isolate human $\mathrm{T}$ cell lymphotropic virus type 1 and to detect variant-specific genomic sequences by polymerase chain reaction in Melanesians with indeterminate Western immunoblot. J Gen Virol 1992;73:1805-10.

14. Gessain A., Mahieux R., De Thé G. HTLV-1 "indeterminate" Western blot patterns observed in sera from tropical regions: the situation revisited (Letter). J Acquir Immune Defic Syndr Hum Retrovirol 1995;9(3):316-8.

15. De Castro-Costa C.M., Goubau P., Liu H.-F., et al. HTLVnegative and HTLV-positive tropical spastic paraparesis in Northeastern Brazil. AIDS Res Hum Retroviruses 1995; $11: 315-18$. 
16. Lal R.B., Rudolph D.L., Coligan J.E., et al. Failure to detect evidence of human T-lymphotropic virus (HTLV) type 1 and type 2 in blood donor with isolated gag antibodies to HTLV-1/2. Blood 1992;80:544-50.

17. Césaire R., Bera O., Maier H., et al. Seroindeterminate patterns and seroconversions to human T-lymphotropic virus type 1 positivity in blood donors from Martinique, French West Indies. Transfusion 1999;39:1145-9.

18. Mauclère P., Le Hesran J.Y., Mahieux R., et al. Demographic, ethnic, and geographic differences between human T cell lymphotropic virus (HTLV) type1-seropositive carriers and persons with HTLV-1 gagindeterminate Western blots in Central Africa. J Infect Dis 1997;176:505-9.

19. Rouet F., Meertens L., Courouble G., et al. Serological, epidemiological, and molecular differences between human T-cell lymphotropic virus Type 1 (HTLV-1)seropositive healthy carriers and persons with HTLV-1 gag indeterminate Western blot patterns from the Caribbean. J Clin Microbiol 2001;39:1247-53.

20. Nogueira C.M., Cavalcanti M., Schechter M., Ferreira O.C. Jr. Human T lymphotropic virus type 1 and 2 infections in healthy blood donors from Rio de Janeiro, Brazil (Letter). Vox Sang 1996;70:47-8.

21. Carneiro-Proietti A.B., Lima-Martins M.V., Passos V.M., et al. Presence of human immunodeficiency virus (HIV) and T-lymphotropic virus type 1 and 2 (HTLV-1/2) in a haemophiliac population in Belo Horizonte, Brazil, and correlation with additional serological results. Haemophilia 1998; 4:47-50.

22. Biggar R.J., Gigase P.L., Melbye M., et al. ELISA HTLV retrovirus antibody reactivity associated with malaria and immune complexes in healthy Africans. Lancet 1985;2:520-3.

23. Yanagihara R., Jenkins C.L., Alexander S.S., et al. Human T-lymphotropic virus type 1 infection in Papua New Guinea: high prevalence among the Hagahai confirmed by Western analysis. J Infect Dis 1990;162:649-54.

24. Hayes C.G., Burans J.P., Oberst R.B. Antibodies to human $\mathrm{T}$ lymphotropic virus type 1 in a population from the Philippines: evidence for cross-reactivity with Plasmodium falciparum. J Infect Dis 1991; $163: 257-62$.

25. Lal R.B., Rudolph D., Alpers M.P., et al. Immunological cross-reactivity between structural protein of human T-cell lymphotropic virus type 1 and the blood stage of Plasmodium falciparum. Clin Diagn Lab Immunol 1994;1:5-10.

26. Porter K.R., Liang L., Long G.W., et al. Evidence for antiPlasmodium falciparum antibodies that cross-react with human T-lymphotropic virus type 1 proteins in a population in Irian Jaya, Indonesia. Clin Diagn Lab Immunol 1994;1:11-5.
27. Porter K.R., Anthony R.L., Solihin A., Hayes C.G. Mapping of a human T-lymphotropic virus type 1 gag protein epitope that cross-reacts with anti-Plasmodium falciparum antibodies. J Med Virol 1995;45:469-74.

28. Sato A., Isaka Y., Morita F., et al. Human sera from varicellazoster virus (VZV) infections cross-react with human T-cell leukaemia virus type 1 (HTLV-1): common epitopes in VZV gene 22 protein and HTLV-1 p19 gag protein. J Gen Virol 1992; 73:2969-73.

29. Banki K., Maceda J., Hurley E., et al. Human T-cell lymphotropic virus (HTLV)-related endogenous sequences, HRES-1, encodes a $28-\mathrm{kDa}$ protein: a possible autoantigen for HTLV-1 gag-reactive autoantibodies. Proc Natl Acad Sci USA 1992;89: 1939-43.

30. Miyakoshi H., Sugimoto M., Igarashi H., et al. Improvement of simultaneous detection of antibodies to gag and envelope antigens of human T-lymphotropic virus type 1 by Western immunoblot assay. J Clin Microbiol 1992;30:2555-59.

31. Segurado A.A., Malaque C.M.S., Sumita L.M., et al. Laboratory characterization of human T-cell lymphotropic virus types 1 (HTLV-1) and 2 (HTLV-2) infections in blood donors from São Paulo (Brazil). Am J Trop Med Hyg 1997;57(2):142-48.

32. Carvalho S.M.F., Pombo-de-Oliveira M.S., Thuler L.C.S., et al. Cross-reactivity between human T-cell leukemia/ lymphoma virus indeterminate Western blot and dengue virus in individuals from Rio de Janeiro, Brazil. J Acquir Immune Defic Syndr Hum Retrovirol 1999;20:4(PO94).

33. Haynes B.F., Robert-Guroff M., Metzgar R.S., et al. Monoclonal antibody against human $\mathrm{T}$ cell leukemia virus p19 defines a human thymic epithelial antigen acquired during ontogeny. J Exp Med 1983;157:907-20.

34. Palker T.J., Singer K.H., Vahlne A. Characterization of an antigen shared by human thymic epithelium and human T cell leukemia virus p19 gag protein. J Acquir Immune Defic Syndr Hum Retrovirol 1996;11:10-19.

35. Soldan S.S., Graf M.D., Waziri A., et al. HTLV-1/2 seroindeterminate Western blot reactivity in a cohort of patients with neurological disease. J Infect Dis 1999; 180: 685-94.

36. Picchio G.R., Bare P., Savignano R., et al. HTLV-1/2 indeterminate serology and natural killer cell expansion. J Acquir Immune Defic Syndr Hum Retrovirol 1996; 12:428-31.

37. Tuppin P., Makuwa M., Guerma T., et al. Low HTLV-1/2 seroprevalence in pregnant women in Congo and a geographic cluster of an HTLV-like indeterminate Western Blot pattern. J Acquir Immune Defic Syndr Hum Retrovirol 1996;11:105-7. 